\title{
Use of a Novel Device in Reconstructive Venous Surgery: Preliminary Results
}

\author{
Daniele Camilli, MD ${ }^{1}$ Alessandro Platone, $\mathrm{MD}^{2} \quad$ Massimo Ruggeri, MD ${ }^{1} \quad$ Sergio Furgiuele, MD ${ }^{1,3}$ \\ ${ }^{1}$ Department of Vascular Surgery, San Camillo de Lellis Hospital, Rieti, \\ Lazio, Italy \\ 2 Department of Vascular Surgery, Isitituto Dermatologio \\ Address for correspondence Daniele Camilli, MD, San Camillo de \\ Lellis Hospital, Viale Kennedy, 02100 Rieti RI, Italy \\ (e-mail: daniele.camilli@gmx.net).
} dell'Immacolata (IDI IRCCS), Roma, Lazio, Italy

${ }^{3}$ Swiss Stem Cell Foundation, Clinical Research, Lugano, Ticino, Switzerland

Int J Angiol 2020;29:245-249.

\begin{abstract}
Evaluation of the outcomes of OSES (oval-shaped external support), a novel device for external valvuloplasty of the great saphenous vein (GSV) for the conservative treatment of superficial venous insufficiency. Between 2012 and 2015, 30 patients underwent external valvuloplasty of the GSV for a total of 32 limbs. Patients were subjected to clinical and instrumental follow-up by a half-year ultrasound for a minimum of 36 months. The main endpoints were the recurrence of varicose disease, persistent or recurrent venous reflux, and venous thrombosis. Varicose recurrence was verified in six limbs on 32 (18.75\%). Four limbs (12.5\%) presented a recurrence of the reflux even in the absence of varicose veins. Two limbs (6.25\%) underwent saphenectomy after the valvuloplasty intervention at 12 and 18 months, respectively, because of the presence of saphenofemoral reflux and varicose

Keywords

- valvuloplasty

- varicose veins

- vein reflux

- venous insufficiency

- vessel repair

- vein patch

- venous ulcer recurrences. No case of venous thrombosis of the saphenous trunk was observed. The external valvuloplasty of the GSV is a well-known technique that used to treat the superficial venous insufficiency. The newly introduced OSES device seems to show better midterm results, due to a better alignment of the valve flaps. In our experience, the use of this device gives better long-term results and allowed to extend the indication to patients with saphenic diameters that were considered not eligible for repair. In conclusion, although our data needs further confirmation, OSES device might represents a new interesting opportunity for reconstructive venous surgery.
\end{abstract}

Chronic venous insufficiency (CVI) is an extremely common disease in the adult population and, in particular, in female population.

The underlying pathogenesis of this disease is not clear. There are different hypotheses, such as the descending, ascending, and microangiopathic theory. Nowadays, none of these pathogenetic theories, relies on a scientific demonstration. In most cases of CVI, the presence of reflux on venous vessels with a profound reversal of normal and physiological vein circulation at the level of the extra fascial vein of the lower limb is observed. ${ }^{1,2}$

The main symptoms of CVI are lower limb heaviness, pain, and skin imperfections. In the most severe cases, cutaneous ulcers, venous thrombosis, and pulmonary embolism may occur.

published online

September 20, 2020
The gold standard of medical therapy are compression and lifestyle improvement in addition to phlebotonic drugs. ${ }^{3}$ Unfortunately, such therapies do not provide any long-lasting healing.

Surgical techniques, used to treat this pathology, can be divided into two main categories: reconstructive and ablative techniques. The ablative techniques include the following: (1) the binding and stripping of the GSV, and (2) the ablation either by endovascular radiofrequency or by laser. Reconstructive techniques consist of hemodynamic reflux correction (ASVAL-CHIVA) and reconstruction of the physiological continence of venous valves by valvuloplasty. The latter technique can be performed both at superficial venous circulation (saphenous vein) level and at deep venous circulation (femoral vein) level. ${ }^{4,5}$ The main limitation of the 10.1055/s-0040-1715132. ISSN 1061-1711. 
devices, currently in use, is the need of ligation of collateral veins at the saphenofemoral junction.

The present study shows the preliminary results of valvuloplasty performed by the use of OSES (oval shaped external support) device.

We found that OSES device allows to restore the physiological valve continence, allowing a better alignment of the valvular cusps, and to preserve the drainage of collateral veins at the same time.

\section{Materials and Methods}

\section{Study Design}

This is a retrospective clinical study, the main endpoints are the improvement of clinical conditions stated by the individual patient, the change in the CEAP score ${ }^{6}$ (- Table 1), recurrence of varicose veins, and need for redo surgery.

\section{Statistical Analyses}

Statistical analyses were performed using InStat and SPSS 20 stat software. Student's $t$-test was used to compare the data collected. A $p$-value $<0.05$ was considered as a significant difference.

\section{Enrollment Criteria}

In this study we included patients with reflux on the terminal or preterminal valve documented by ultrasound examination with morphologic study (valves visible, mobile, and not prolapsed) and with measures of the saphenofemoral junction (-Fig. 1); all patients underwent a follow-up at 36 months; age: young adult.

\section{Exclusion Criteria}

We excluded patients without saphenous reflux or without terminal o preterminal valve (valve's flaps were nonvisible at the ultrasound examination), with segmental reflux of the GSV or with reflux only on collaterals/tributary branches, and with low life expectancy.

\section{Patients Data}

Between 2012 and 2015, 30 patients underwent external valvuloplasty of the great saphenous vein for a total of 32 limbs.

Table 1 Definitions for CEAP clinical grade
CEAP score was used to assess the severity of the pathology before and after the operation. UltraSoundcolor Flow was used to assess the competence of the valves. All patients were evaluated by ultrasound examination before the operation to analyze the hemodynamic asset and to measure the vein diameter to select the device size.

The presence of a reflux more than 0.5 seconds at the level of the valve was considered pathologic and an indication for surgical intervention.

All patients underwent clinical and ultrasound examination follow-up at every 6 months for a mean time of 36 months. At the end of the study, all patients filled in a questionnaire for the clinical outcome evaluation.

\section{Procedural Data}

Intervention was performed under local anesthesia in selected cases under pharmacological sedation. The saphenofemoral junction was isolated together with the affluent collateral veins. The OSES (- Fig. 2) device has been positioned around the GSV and sutured with prolene 6.0 at the level of the valve (-Fig. 3). Exact position of the valve cusps can be located visually by the operator according to the preoperative distance from the common femoral vein measured with the ultrasound. The procedure was completed by flebectomy of the tributary veins according to preoperative hemodynamic strategy. Patients were discharged with elastic bandage that was removed after 7 days. The use of an elastic sock (compression class 1) was suggested for at least 6 months.

\section{Results}

Among 30 patients enrolled, 24 were female (80\%) and 6 were male (20\%), with a mean age of 43.5 years (minimum, 28 years and maximum, 65 years). Dyslipidemia, hypertension, and dysthyroidism were the most common comorbidities.

There were no intraoperative or postoperative complications.

No cases of deep venous thrombosis were observed, and no one complained about any symptom related to the device implanted (neither rejection syndrome nor pain at the groin were observed).

At the end of the study (36 months), 26 (81.25\%) limbs showed a complete restoration of the valvular competence with the abolition of the reflux and no visible varices. Two

\begin{tabular}{|l|l|l|}
\hline \multicolumn{2}{|l|}{ CEAP clinical grade } & No visible or palpable varicose veins \\
\hline C0 & & Teleangectasia (thread veins/spider veins/broken veins) \\
\hline C1 & C2A & Varicose veins without any symptoms (asymptomatic) \\
\cline { 2 - 3 } & C2B & Varicose veins with symptoms \\
\hline C3 & & Swollen ankle (edema) due to varicose veins or hidden varicose veins (venous reflux) \\
\hline C4 & & Skin damage due to varicose veins of hidden varicose veins (venous reflux) \\
\hline C5 & & Healed venous leg ulcer \\
\hline C6 & & Venous leg ulcer \\
\hline
\end{tabular}

Abbreviation: CEAP, clinical-etiological-anatomical-pathophysiological. 


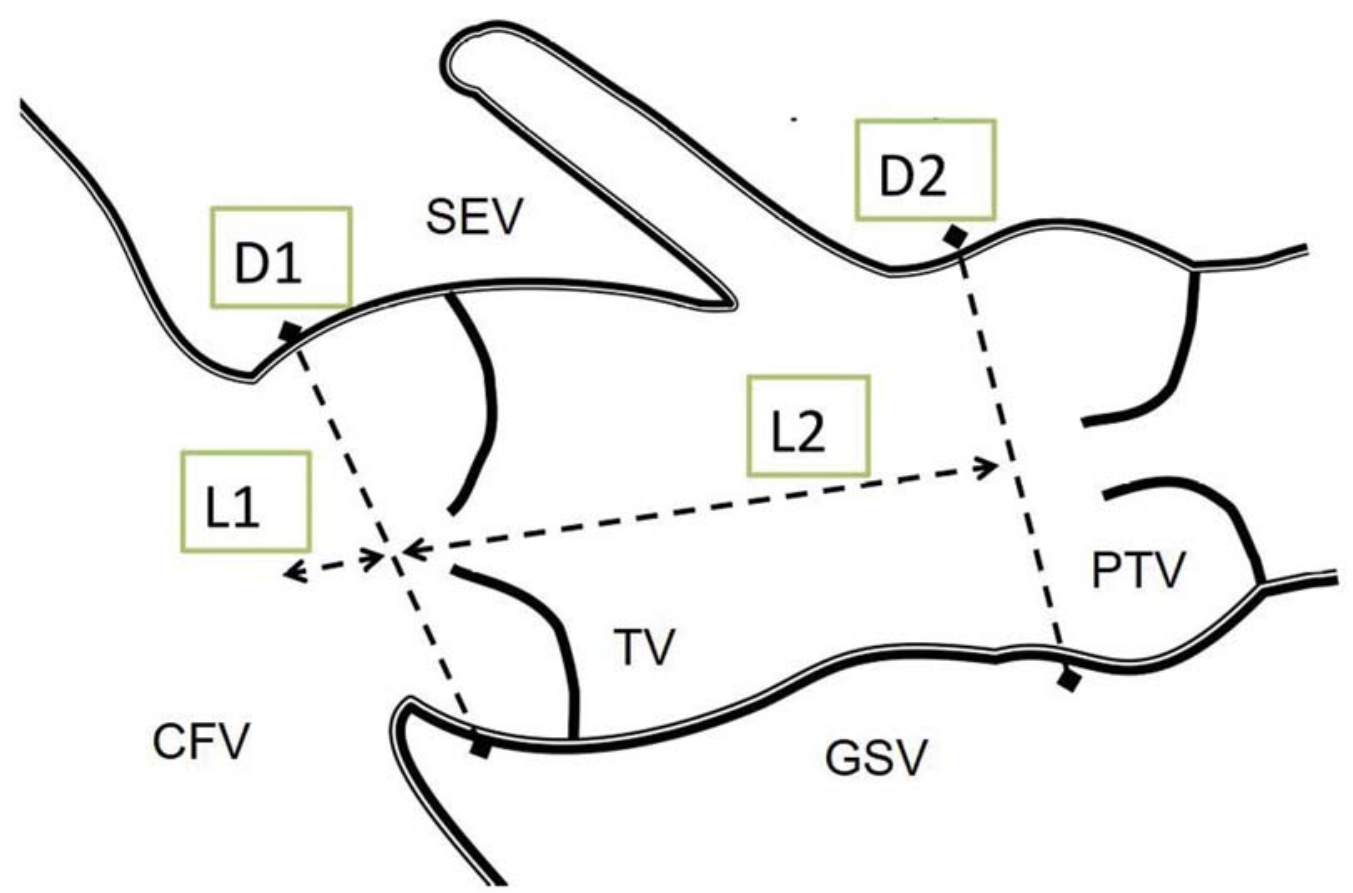

Fig. 1 Preoperative morphologic and measure record. CFV, common femoral vein; D1, TV diameter; D2, PTV diameter; GSV, great saphenous vein; L1, distance from GSV origin and TV; L2, distance from GSV origin and PTV; PTV, preterminal valve; SEV, superficial eipgastric vein; TV, terrminal valve.

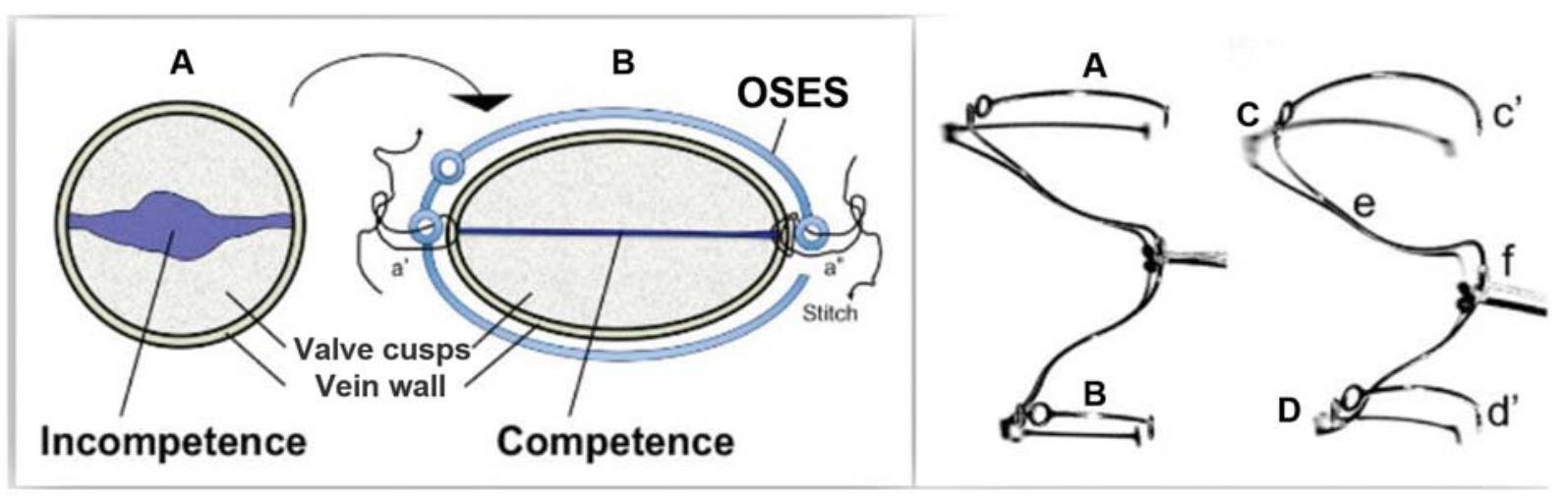

Fig. 2 OSES device working principle. Left: Great saphenous vein section before (A) and after (B) the position of the OSES device; a"1 and a" ${ }^{2}$ prolene stitches. Right: the OSES device: (A) proximal stent; (C) and C1 holes to suture the device at the level of the terminal valve; (B) distal stent; (D) and D1 holes to suture the device at the level of the preterminal valve. e, support stent; $f$, strings used to help the positioning of the device and then removed; OSES, oval-shaped external support.

(6.25\%) limbs showed a low reflux ( $>0.5$ and $<1$ seconds) and four (12.5\%) showed the presence of long reflux ( $>1$ second). In two (6.25\%) cases, it was necessary to remove the device and perform GSV stripping, at 12 and 18 months after the intervention, respectively, because of the presence of reflux and worsening of symptoms (-Table $\mathbf{2}$ ).

The patient-standing GSV mean diameter decreased from 8.32 to $6.80 \mathrm{~mm}$ at terminal valve level and from 7.34 to $5.71 \mathrm{~mm}$ at the preterminal valve level. A lower difference was observed when GSV mean diameter was measured with the patient supine $(6.85-5.72 \mathrm{~mm}$ at terminal valve level; $5.97-4.69 \mathrm{~mm}$ at preterminal valve level). In three cases, there was no significant change in diameter before and after the operation. In two cases, no comparison was possible due to the removal of the device ( - Table 3 ).
All patients were classified as CEAP C2s preoperatively. At the end of follow-up, seven (23.3\%) patients had recurrence varices but didn't necessitate a second surgery (CEAP C2a). The mean venous clinical severity score $(\text { VCSS })^{6}$ was $6.52 \pm 0.58$ preoperatively and $1.9 \pm 0.88$ at the end of the follow-up.

\section{Discussion}

Varicose disease is a frequent cause of ulcerative disease in the lower limbs, ${ }^{2,7}$ phlebitis, or deep thrombosis and it affects quality of life. The main problem of the current therapeutic approach is the high number of recurrences following traditional ablative surgery (stripping or endovascular surgery). Hemodynamic treatment with saphenous trunk was shown to have a lower percentage of long-term recurrence. ${ }^{8,9}$ 


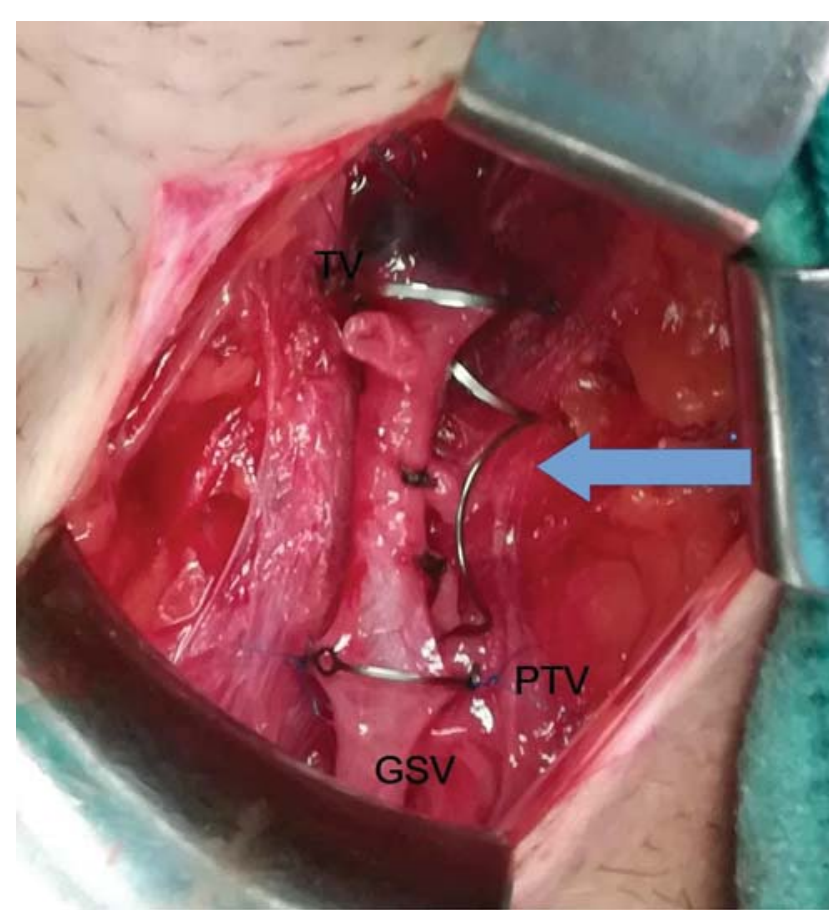

Fig. 3 OSES device in situ (arrow); GSV, great saphenous vein; OSES, oval-shaped external support; PTV, preterminal; TV, terrminal valve.

The reasons of recurrences are not fully understood. Often there is neovascularization following surgery at the groin level. In other cases, there is evidence of varicose recurrences at the leg level that show chaotic circulation without a clear hemodynamic rationale.

Some authors have suggested that one of the causes of this recurrence is due to the lack of a drainage vessel (the GSV) that, when correctly continent, decreases the blood volume drained by the deep venous circulation. ${ }^{10}$ The absence of this drainage pathway could lead to femoral venous hypertension and to the need to establish low flow channels able to support the function of the femoral vein. In many cases, the amount of blood volume exceeds the drainage capacity of the deep venous system and burdens on the superficial venous circulation. Such blood flow, without a drainage pathway, would result in venous wall changes and, ultimately, venous dilation visible as varices.

Following these observations, reconstitution of physiological valve continence would be a valid therapeutic approach to decrease the rate of varicose recurrence. Preserving the main superficial drainage vessel (the GSV) shortens, the volume of blood that is directed to the level of the deep venous circulation. The presence of competent valves also reduce the weight of the hydrostatic column and produce a drainage effect on the tributary collateral vessels.

Hemodynamic effects of valve competence restoration are current subject of study, but clinical results suggest that the presence of auxiliary drainage pathway to the femoral vein is a protective element and can reduce the risk of varicose recurrences.

The OSES device, unlike other devices or valvuloplasty techniques (plication, trapdoor internal valvuloplasty, ${ }^{11}$ etc.), acts by stretching on the valve cusps and not by generating pressure from the outside (e.g., "Gore" external valve support ${ }^{12}$ ). In our opinion, this type of mechanism allows a better efficacy and a better result in terms of valve competence, as evidenced by the ultrasound control when traction is performed exactly at the level of the valve joint.

The structure of the device also saves all the drainage vessels at the level of the femoral artery (epigastric, circonflex, pudenda vein, etc.), helping the femoral vein drainage. The ultimate goal is to "rebuild" the original, physiological, and sophisticated design of the saphenofemoral junction, directing the stream in the right way toward the iliac circulation and, finally, reducing the hemodynamic pressure on the superficial venous compartment.

Short-term results were good. In $81.25 \%$ of cases, terminal valve was continent at 1 year follow-up, the amount of reinterventions was low (6.25\%) with a low-recurrence rate (18.75\%). Only in two cases, the GSV stripping was performed

Table 2 Clinical results on 32 limbs' ultrasound evaluation of anatomical modifications

\begin{tabular}{|c|c|c|c|c|c|c|c|c|}
\hline \multirow[t]{2}{*}{ Clinical results on 32 limbs } & \multicolumn{2}{|c|}{ Preoperative } & \multicolumn{6}{|l|}{ Follow-up } \\
\hline & $n$ & $\%$ & 1 month & $\%$ & 1 year & $\%$ & 3 year & $\%$ \\
\hline Terminal valve reflux (no varices) & 0 & 0,00 & 10 & 31.3 & 4 & 12.50 & 4 & 12.50 \\
\hline Terminal valve reflux (and varices) & 32 & 100.00 & 0 & 0.0 & 1 & 3.13 & 2 & 6.25 \\
\hline Preterminal valve reflux & 0 & 0.00 & 0 & 0.0 & 0 & 0.00 & 0 & 0.00 \\
\hline Tributaries & 32 & 100.00 & 4 & 12.5 & 6 & 18.75 & 6 & 18.75 \\
\hline CEAP C2a & 0 & 0.00 & 4 & 12.5 & 4 & 12.50 & 4 & 12.50 \\
\hline CEAP C2s & 32 & 100.00 & 0 & 0.0 & 1 & 3.13 & 1 & 3.13 \\
\hline CEAP C4 & 0 & 0.00 & 0 & 0.0 & 0 & 0.00 & 0 & 0.00 \\
\hline VCSS (mean) & 6.5 & & 1.22 & & 1.96 & & 1.96 & \\
\hline Deep venous thrombosis & 0 & 0.00 & 0 & 0.0 & 0 & 0.00 & 0 & 0.00 \\
\hline Superficial venous thrombosis & 1 & 3.13 & 0 & 0.0 & 0 & 0.00 & 0 & 0.00 \\
\hline Further surgery & 0 & 0.00 & 0 & 0.0 & 1 & 3.13 & 2 & 6.25 \\
\hline
\end{tabular}

Abbreviations: VCSS, venous clinical severity score; CEAP, Clinical-Etiological-Anatomical-Pathophysiological score. 
Use of a Novel Device in Reconstructive Venous Surgery Camilli et al. 249

Table 3 UltraSound evaluation of anatomical modifications (mean values in mm and SD)

\begin{tabular}{|l|c|c|c|c|}
\hline & $\begin{array}{c}\text { Preoperative } \\
\text { (mm and SD) }\end{array}$ & $\begin{array}{c}\text { Follow-up } \\
\text { (mm and SD) }\end{array}$ & Difference (mm) & $p$ \\
\hline $\begin{array}{l}\text { Mean distance from GSV origin and } \\
\text { terminal valve (mm) }\end{array}$ & $3.95 \pm 2.60$ & $4.82 \pm 1.60$ & 0.87 & 0.30 \\
\hline $\begin{array}{l}\text { Mean distance from GSV origin and } \\
\text { preterminal valve (mm) }\end{array}$ & $19.74 \pm 7.10$ & $16.66 \pm 6.80$ & -3.08 & 0.20 \\
\hline $\begin{array}{l}\text { Mean terminal valve diameter: } \\
\text { patient upright (mm) }\end{array}$ & $8.32 \pm 3.70$ & $6.80 \pm 1.70$ & -1.52 & 0.40 \\
\hline $\begin{array}{l}\text { Mean terminal valve diameter: } \\
\text { patient supine (mm) }\end{array}$ & $6.85 \pm 3.20$ & $5.72 \pm 1.70$ & -1.13 & 0.10 \\
\hline $\begin{array}{l}\text { Mean preterminal valve diameter: } \\
\text { patient upright (mm) }\end{array}$ & $7.34 \pm 3.00$ & $5.71 \pm 2.00$ & -1.63 & 0.30 \\
\hline $\begin{array}{l}\text { Mean preterminal valve diameter: } \\
\text { patient supine (mm) }\end{array}$ & $5.97 \pm 3.20$ & $4.69 \pm 1.60$ & 1.28 & \\
\hline
\end{tabular}

Abbreviations: GSV, great saphenous vein; SD, standard deviation.

that even after valvuloplasty remains a therapeutic opportunity that may, if necessary, still be performed. Randomized studies showed that hemodynamic treatment (CHIVA) has better long-term results than stripping. Stripping and endovascular ablation give comparable results but the rate of recurrences or reinterventions is still not negligible. According to these studies, hemodynamic treatment associated with reconstruction of valve continence may be a valid strategy for achieving the best long-term outcome.

\section{Conclusion}

Valvular incompetence is a frequent occurrence in patients with chronic venous insufficiency. In many studies, it has been highlighted that valvuloplasty is an effective technique in correcting this situation by reconstructing the normal and physiological valve function. In the case of young patients with mobile valve cusps, this type of approach can be a valid alternative to traditional ablation surgery that causes complete subversion of physiological venous hemodynamics of the lower limb. Reconstruction of normal venous circulation could reduce or reset the tendency to distance relapse. The OSES device has been proved to be technically easy to use, restores the valve competence, and saves the drainage given by the tributaries at the saphenofemoral junction. Midterm results were positive in terms of reflux elimination and of clinical condition improvement. The results of varicose relapse reduction are currently being studied and will be evaluated over time.

\section{Conflict of Interest}

None declared.

\section{References}

1 Perrin M, Eklof B, VAN Rij A, et al. Venous symptoms: the SYM Vein Consensus statement developed under the auspices of the European Venous Forum. Int Angiol 2016;35(04):374-398

2 Vaidyanathan S, Menon RR, Jacob P, John B. Chronic Venous Disorders of the Lower Limbs: A Surgical Approach. India: Springer; 2014

3 Appelen D, van Loo E, Prins MH, Neumann MH, Kolbach DN. Compression therapy for prevention of post-thrombotic syndrome. Cochrane Database Syst Rev 2017;9:CD004174

4 Kistner RL. Surgical repair of the incompetent femoral vein valve. Arch Surg 1975;110(11):1336-1342

5 Belcaro GV. Plication of the sapheno-femoral junction: effects on incompetence after two years. Phlebology 1991;6(03):159-165

6 Kakkos SK, Rivera MA, Matsagas MI, et al. Validation of the new venous severity scoring system in varicose vein surgery. J Vasc Surg 2003;38(02):224-228

7 Marola S, Ferrarese A, Solej M, Enrico S, Nano M, Martino V. Management of venous ulcers: state of the art. Int J Surg 2016;33 (Suppl 1):S132-S134

8 Carandina S, Mari C, De Palma M, et al. Varicose vein stripping vs haemodynamic correction (CHIVA): a long term randomised trial. Eur J Vasc Endovasc Surg 2008;35(02):230-237

9 Parés i Rifà JO. Randomised controlled trial of surgical treatment of varicose veins in legs: Stripping versus Conservative Treatment (CHIVA). 2012. Doi: 10.1186/isrctn52861672

10 Pittaluga P, Chastanet S, Rea B, Barbe R. Méthode ASVAL (ablation sélective des varices sous anesthésie locale): principes, technique, indications et expérience personnelle. J Mal Vasc 2007;32:4. 10.1016/j.jmv.2007.01.062

11 Tripathi R, Ktenidis KD. Trapdoor internal valvuloplasty-a new technique for primary deep vein valvular incompetence. Eur J Vasc Endovasc Surg 2001;22(01):86-89

12 Sarac A, Jahollari A, Talay S, Ozkaya S, Ozal E. Long-term results of external valvuloplasty in adult patients with isolated great saphenous vein insufficiency. Clin Interv Aging 2014;9:575-579 